\title{
Effet du sens du croisement sur la teneur et la composition chimique des huiles essentielles des différents hybrides d'Eucalyptus cultivés au Maroc
}

\author{
Abdellah Farah ${ }^{\mathrm{a}, \mathrm{b}^{*}}$, Mohamed Fechtal $^{\mathrm{a}}$ and Abdelaziz Chaouch ${ }^{\mathrm{b}}$ \\ ${ }^{a}$ Centre National de la Recherche Forestière, BP 763, Rabat Agdal, 10 050, Maroc

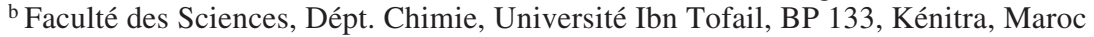

(Reçu le 24 juillet 2000 ; accepté le 21 septembre 2001)

\begin{abstract}
Résumé - Partant des espèces parentales E. camaldulensis, E. grandis et E. tereticornis, nous avons étudié l'effet des croisements, dans les deux sens, sur la teneur et la composition chimique des huiles essentielles des hybrides. La teneur, par rapport au poids sec, en huiles essentielles des espèces parentales : E. camaldulensis, E. grandis et E. tereticornis sont respectivement 1, 0,37 et $0,82 \%$. L'hybride $E$. grandis $(q) \times$ E. camaldulensis $(0)$ fournit une teneur moyenne de $0,80 \%$ contre $0,70 \%$ pour le croisement réciproque. Les échantillons de l'hybride $E$. tereticornis $\times$ E. camaldulensis fournissent une teneur moyenne de $0,92 \%$, le croisement réciproque donne une teneur de $0,84 \%$. L' $\alpha$-pinène, le p-cymène et le 1,8-cinéole constituent les principaux composés majoritaires de ces huiles essentielles. Le taux de ces composés varie selon les espèces parentales mais aussi, selon le sens du croisement des hybrides. Ces valeurs sont souvent intermédiaires entre celles des parents, mais en aucun cas supérieures à celles des échantillons du parent dont le taux est le plus élevé. Les résultats de la composition chimique des différents échantillons étudiés sont discutés. Aucune des combinaisons ne permet d'atteindre les teneurs en 1,8-cinéole suffisants pour répondre à la norme en vigueur.
\end{abstract}

E. camaldulensis / E. grandis / E. tereticornis / hybrides / croisement / croisement réciproque / huiles essentielles / rendement / composition chimique

Summary - Effect of crossing and reciprocal crossing on the content and the chemical composition of essential oils of Eucalyptus hybrid growing in Morocco. From parental species E. camaldulensis, E. grandis and E. tereticornis we have studied the effect of crossing and reciprocal crossing on yield and chemical composition of essential oils of eucalyptus hybrids. The E. camaldulensis, E. grandis and E. tereticornis yields were respectively $1,0.37$ and $0.82 \%$. The hybrid E. grandis $(\$) \times E$ camaldulensis $(\stackrel{\$}{)})$ give an average yield of about $0.8 \%$ against $0.7 \%$ for his reciprocal crossing. The eucalyptus hybrid E. tereticornis $\times$ E. camaldulensis yield is about $0.9 \%$, the reciprocal crossing yield is about $0.84 \%$. The $\alpha$-pinene, p-cymene and 1,8-cineole are principal compounds of these essential oils. The percentage of these products vary according to parentals species and the crossing sens. These values take often intermediate values compared to parental species, but not higher than those of the parents. The results on chemical composition from different samples are discussed. However, no combination attains a sufficient content of 1,8-cineole to fit to the usual norm.

E. camaldulensis / E. grandis / E. tereticornis / hybrid / crossing / reciprocal crossing / essential oils / yield / chemical composition

* Correspondance et tirés-à-part :

Tél. : 21237673830 ; fax : 21237671151 ; e-mail : farah117@ caramail.com 


\section{INTRODUCTION}

Originaires d'Australie, les eucalyptus ont été introduits et plantés, avec un très grand succès au Maroc. Les espèces : Eucalyptus camaldulensis et E. gomphocephala ont connu une extension relativement importante. Actuellement, la superficie occupée par les plantations d'eucalyptus est estimée à 202000 ha dont environ 44 et $31 \%$ correspondent respectivement à E. camaldulensis et $E$. gomphocephala [12]. Le bois de ces espèces est essentiellement destiné à la trituration. En dehors de ces deux espèces, un certain nombre d'hybrides ont été mis au point. L'objectif visé est de sélectionner des hybrides (naturels ou artificiels) capables de répondre aux critères d'adaptation, de résistance, et de qualité de bois de trituration $[7,10,14]$. Par ailleurs, dans le cadre de la valorisation de la biomasse foliaire de ces hybrides, une étude sur les huiles essentielles de cette matière première a été menée [8]. En effet, les huiles essentielles d'E. camaldulensis, espèce la plus importante dans les reboisements, présentent un taux de 1,8-cinéole, le principale principe actif recherché par les utilisateurs, inférieur à $70 \%$ [20, 21], taux exigé par la norme en vigueur [17]. En raison de ses exigences climatiques et édaphiques E. globulus, espèce très demandée pour la qualité de ses huiles essentielles dans le marché international [1], ne peut être plantée que dans la région côtière Nord-Ouest du Maroc [9]. Il est donc nécessaire de chercher des hybrides susceptibles de répondre aux exigences du marché. Par ailleurs au Congo, les études comparatives effectuées sur des hybrides apparus naturellement, tel que l'hybride $E$. europhylla $\times E$. grandis, ont révélé l'influence de l'hybridation sur les caractéristiques chimiques des huiles essentielles et la transmission biparentales de ces caractéristiques $[5,11]$.

Au cours de cette étude, diverses combinaisons génétiques à partir des espèces $E$. camaldulensis var. albakutia, E. grandis Hill. ex. Maid. et E. tereticornis Sm. ont été étudiées. L'effet des croisements dans les deux sens entre espèces parentales sur la quantité et la qualité des huiles essentielles a été déterminé.

\section{MATÉRIEL ET MÉTHODES}

\subsection{Matériel végétal}

Au cours de ce travail, les échantillons de quatre combinaisons à base des espèces E. camaldulensis, E. grandis et E. tereticornis ont été récoltés dans un dispositif en blocs aléatoires complet selon Wright [18], constitué de
6 répétitions de parcelles linéaires de 5 plants. Les combinaisons génétiques analysées sont présentées dans l'ordre génétique «E. femelle $\times$ E. mâle » :

E. camaldulensis $\times$ E. grandis ;

E. grandis $\times$ E. camaldulensis ;

E. camaldulensis $\times$ E. tereticornis ;

E. tereticornis $\times$ E. camaldulensis.

Ces hybrides ont été créés par pollinisation contrôlée, au cours d'un programme d'amélioration génétique des eucalyptus au Maroc [7, 14]. Les échantillons de ces hybrides proviennent du dispositif expérimental de Mechraâ El Kettane situé dans la Mamora occidentale. Ceux des espèces parentales, proviennent du verger d'hybridation de Sidi Amira, installé par greffage sur des portegreffes compatibles en 1988. Ces dispositifs bénéficient de conditions climatiques et édaphiques similaires [2]. Trois arbres ont été choisis au hasard pour chaque espèce parentale $(3 \times 3)$ et pour chaque combinaison $(4 \times 3)$, soit un total de 21 arbres échantillons. Les plantations de ces hybrides ont été réalisées en 1996 (essais de descendance). Les feuilles ont été récoltées au mois de juin 1998. Au cours de ces essais, seules les feuilles adultes ont été distillées.

\subsection{Extraction des huiles essentielles}

L'extraction des huiles essentielles a été effectuée par un appareil de distillation de type Clevenger [6], constitué d'un ballon de un litre surmonté par une colonne de $45 \mathrm{~cm}$ de hauteur. La distillation de chaque échantillon dure environ trois heures $[8,19]$. Ce type d'extraction est le plus souvent employé pour l'extraction des huiles essentielles des plantes aromatiques.

Dans chaque essai d'extraction, 200 à $250 \mathrm{~g}$ de feuilles adultes ont été traitées. Trois extractions au moins ont été réalisées pour chaque arbre échantillon, soit un total de 72 extractions. Les huiles essentielles sont soumises aux analyses chromatographiques. Auparavant, l'humidité d'un échantillon représentatif de la biomasse foliaire a été déterminée par étuvage à $60{ }^{\circ} \mathrm{C}$ jusqu'à poids constant. Les teneurs moyennes en huiles essentielles ont été calculées par rapport à la matière sèche et exprimées en $\mathrm{ml} / 100 \mathrm{~g}$. L'analyse de variance a été effectuée par le logiciel Systat 7.2.

\subsection{Analyses chromatographiques}

Les analyses chromatographiques ont été effectuées sur un chromatographe en phase gazeuse à régulation électronique de pression de type Hewlett-Packard (série HP 6890), équipé d'une colonne capillaire HP-5 
$(30 \mathrm{~m} \times 0,25 \mathrm{~mm})$ avec une épaisseur du film de $0,25 \mu \mathrm{m}$, d'un détecteur FID alimenté par un mélange de gaz $\mathrm{H}_{2} /$ Air et d'un injecteur split - splitless. La température du détecteur est de $260{ }^{\circ} \mathrm{C}$, celle de l'injecteur est de $275{ }^{\circ} \mathrm{C}$. Le gaz vecteur utilisé est l'azote avec un débit de $2 \mathrm{ml} / \mathrm{min}$. La température de la colonne est programmée de 50 à $250^{\circ} \mathrm{C}$ à raison de $4{ }^{\circ} \mathrm{C} / \mathrm{min}$. L'appareil est piloté par un système informatique de type "HP ChemStation ».

L'identification des constituants a été réalisée en se basant sur leurs Indices de Kovats (IK) et sur la chromatographie en phase gazeuse couplée à la spectrométrie de masse (GC-MS). Cette dernière est réalisée sur un chromatographe en phase gazeuse de type Hewlett-Packard (série HP 6890) couplé avec un spectromètre de masse (série HP 5973). La fragmentation est effectuée par impact électronique sous un champ de $70 \mathrm{eV}$. La colonne utilisée est une colonne capillaire DB-1 $(50 \mathrm{~m} \times 0,25 \mathrm{~mm})$, l'épaisseur du film est de $0,25 \mu \mathrm{m}$. Le gaz vecteur est l'hélium dont le débit est fixé à $1,7 \mathrm{ml} / \mathrm{min}$. La température de la colonne est programmée de 65 à $250{ }^{\circ} \mathrm{C}$ à raison de $2{ }^{\circ} \mathrm{C} / \mathrm{min}$. L'appareil est relié à un système informatique gérant une bibliothèque de spectres de masse NBS $75 \mathrm{KL}$ et une bibliothèque interne spécifique des arômes réalisée par le laboratoire interrégional de Marseille (France).

\section{RÉSULTATS ET DISCUSSION}

\subsection{Teneurs en huiles essentielles}

Les teneurs en huiles essentielles varient d'une espèce à l'autre et l'analyse de variance à un seul critère de clas sification montre qu'il y a une différence hautement significative entre les teneurs en huiles essentielles des différents échantillons étudiés; $F_{0,95}$ obs. $=119$ (tableaux $I$ et II). Les échantillons d'E. camaldulensis fournissent les teneurs les plus élevées, en moyenne $1 \%$ contre $0,82 \%$ pour E. tereticornis et $0,37 \%$ pour E. grandis. Ces résultats confirment ceux qui ont été rapportés par la littérature $[8,20,21]$.

La comparaison des teneurs moyennes obtenues à partir de la biomasse foliaire des espèces parentales $E$. $c a$ maldulensis, E. grandis et de leurs hybrides E. camaldulensis $\times E$. grandis et E. grandis $\times E$. camaldulensis montre, d'après l'analyse de variance à un seul critère de

Tableau I. Teneurs en huiles essentielles des feuilles par rapport à la matière sèche.

\begin{tabular}{|c|c|c|}
\hline Échantillons & Teneur moyenne $(\mathrm{ml} / 100 \mathrm{~g})$ & Coefficients de Variation CV (\%) \\
\hline E. camaldulensis ( $\mathrm{ca}$ ) & 1,00 & 2,00 \\
\hline E. grandis (gr) & 0,37 & 12,39 \\
\hline E. tereticornis (te) & 0,82 & 3,66 \\
\hline E. $c a \times E . g r$ & 0,70 & 7,14 \\
\hline E. $g r \times E . c a$ & 0,80 & 5,00 \\
\hline E. $c a \times$ E.te & 0,84 & 7,78 \\
\hline E.te $\times$ E.ca & 0,92 & 5,60 \\
\hline
\end{tabular}

E. ca $\times$ E. gr $:$ E. camaldulensis $\times$ E. grandis, E. ca $\times$ E. te $:$ E. camaldulensis $\times$ E. tereticornis.

$\mathrm{CV}(\%)=$ coefficient de variation (Écart type/ moyenne) $\times 100$.

Tableau II. Analyse de variance à un seul critère de classification (effet des espèces et de leurs hybrides sur la teneur en huiles essentielles).

\begin{tabular}{lllll}
\hline Source & Somme des carrés des écarts & Degré de liberté & Carrés moyens & $F_{\text {obs }}$ \\
\hline Espèces & 0,991 & 6 & 0,165 & $119^{* * *}$ \\
Erreur & 0,029 & 21 & 0,001 & \\
\hline
\end{tabular}

*** : hautement significatif. 
classification, une différence hautement significative (tableau IIIa). La biomasse foliaire des hybrides donne des teneurs intermédiaires à ceux de la biomasse foliaire de leurs espèces parentales, ces teneurs restant inférieures à celle du parent $E$. camaldulensis. Les échantillons de l'hybride $E$. grandis $\times E$. camaldulensis fournissent une teneur moyenne en huiles essentielles légèrement supérieure à celle du croisement réciproque, soit respectivement 0,80 et $0,70 \%$. Ces résultats confirment ce qui a été avancé par la littérature $[13,16]$.

L'analyse de variance à un seul critère de classification montre également qu'il y a une différence significative entre les espèces parentales $E$. camaldulensis, E. tereticornis et leurs hybrides E. camaldulensis $\times$ E. tereticornis et E. tereticornis $\times E$. camaldulensis $\left(F_{0,95}\right.$ obs. $\left.=20\right)($ tableau IIIb). Les teneurs obtenues au cours de ces essais varient d'une combinaison à l'autre. La meilleure teneur a été obtenue avec les échantillons de l'hybride E. tereticornis $\times$ E. camaldulensis, soit une moyenne d'environ $0,92 \%$, la teneur moyenne du croisement réciproque étant de $0,84 \%$. Cette teneur est donc inférieure à celle de la biomasse foliaire du parent femelle E. camaldulensis $(1 \%)$ et sensiblement égale à celle des feuilles du parent mâle E. tereticornis $(0,82 \%)$.

\subsection{Composition chimique des huiles essentielles}

Les analyses chromatographiques des huiles essentielles ont permis de détecter et d'identifier une cinquantaine de constituants. Le tableau IV regroupe les principaux de ces constituants, composés habituels des huiles essentielles des eucalyptus $[3,4,15,19,20]$.
Les échantillons de biomasse foliaire des espèces parentales E. camaldulensis, E. grandis et E. tereticornis présentent des huiles essentielles avec l' $\alpha$-pinène, le pcymène, et le 1,8-cinéole comme constituants majoritaires (tableau IV). En plus de ces produits, les huiles essentielles d'E. camaldulensis sont caractérisées par la présence de l' $\alpha$-terpinéol (4,58\%) et de carvone $(3,60 \%)$. Les huiles essentielles d'E. grandis révèlent l'existence d'autres produits tels que le $\alpha$-terpinène $(3,30 \%)$, le fenchol $(3,85 \%)$, le trans pinocarvéol (6,64\%), l' $\alpha$-terpinéol $(4,94 \%)$ et le globulol $(5,34 \%)$. Le cryptone, produit caractéristique des huiles essentielles d'E. tereticornis, est détecté avec un taux d'environ 4,60\%. Les travaux effectués par Chalchat et al. [4] sur les échantillons de la même espèce acclimatée au Ruanda, ont démontré que le cryptone caractérise ses huiles essentielles avec environ $11 \%$. Les huiles essentielles de cette espèce présentent, également, le $\alpha$-terpinène, l'aromadendrène et l'allo-aromadendrène avec des teneurs moyennes respectives de $4 \%, 3 \%$ et $10 \%$ (tableau IV). Le meilleur taux de 1,8-cinéole est obtenu avec les huiles essentielles d'E. camaldulensis avec environ $50 \%$. Cette valeur est supérieure à celles obtenues avec les espèces E. tereticornis et $E$. grandis, soit des teneurs respectives de 31 et $21 \%$. Cependant ces taux sont inférieurs au seuil minimum de $70 \%$ exigé par l'industrie pharmaceutique [17].

L'examen de la composition chimique des huiles essentielles de la biomasse foliaire des hybrides indique que l'hybridation n'a pas favorisé la biosynthèse de nouveaux produits (tableau IV). Les taux des différents constituants provenant des hybrides prennent des valeurs intermédiaires par comparaison avec celles des huiles

Tableau IIIa. Analyse de variance à un seul critère de classification (effet des espèces E. camaldulensis, E. grandis et leurs hybrides sur la teneur en huiles essentielles).

\begin{tabular}{lllll}
\hline Source & Somme des carrés des écarts & Degré de liberté & Carrés moyens & $F_{\text {obs· }}$ \\
\hline Espèces & 0,831 & 3 & 0,277 & $251,7 * * *$ \\
Erreur & 0,013 & 12 & 0,001 & \\
\hline
\end{tabular}

*** : hautement significatif.

Tableau IIIb. Analyse de variance à un seul critère de classification (effet des espèces E. camaldulensis, E. tereticornis et leurs hybrides sur la teneur en huiles essentielles).

\begin{tabular}{lllll}
\hline Source & Somme des carrés des écarts & Degré de liberté & Carrés moyens & $F_{\text {obs }}$. \\
\hline Espèces & 0,082 & 3 & 0,027 & $20,02 * *$ \\
Erreur & 0,016 & 12 & 0,001 &
\end{tabular}

** : significatif. 
essentielles des feuilles de leurs espèces parentales, ce qui rejoint les résultats de travaux rapportés par la littérature sur d'autres hybrides $[13,16]$.

L'analyse de variance à un seul critère de classification montre qu'il y a une différence hautement significative (tableau $V$ ) entre les taux de 1,8-cinéole des huiles essentielles des différents échantillons étudiés. Les huiles essentielles des feuilles du croisement E. grandis $\times$ E. camaldulensis et celles du croisement réciproque E. camaldulensis $\times E$. grandis présentent des taux de 1,8-cinéole très proches, soit respectivement 46 et $48 \%$ (tableau IV et figure 1). Ces valeurs sont deux

Tableau IV. Composition chimique des huiles essentielles des hybrides et des espèces parentales d'Eucalyptus exprimée en pourcentage du total des huiles essentielles.

\begin{tabular}{|c|c|c|c|c|c|c|c|c|}
\hline I.K & Composés & E. $c a$ & E. $g r$ & E.te & E. $c a \times E . g r$ & E. $g r \times E . c a$ & E. $c a \times$ E. te & E. te $\times$ E. ca \\
\hline 939 & $\alpha$-pinène & 11,23 & 14,64 & 7,02 & 14,1 & 12 & $\mathbf{8 , 8 7}$ & 7,68 \\
\hline 952 & Camphène & - & 2,6 & 0,2 & 0,04 & 2,82 & 0,17 & 0,04 \\
\hline 980 & $\alpha$-pinène & 1,88 & 0,12 & 0,21 & 0,11 & 0,48 & 0,15 & 0,26 \\
\hline 1006 & $\alpha$-phellandrène & 0,18 & 0,23 & 0,15 & 0,12 & 0,26 & 0,10 & 0,3 \\
\hline 1026 & p-cyméne & 11,24 & 23,20 & 5,30 & 18,64 & 8,5 & 6,30 & 10,6 \\
\hline 1031 & Limonène & 1,11 & - & 4,12 & - & 1,60 & 1,30 & 2,00 \\
\hline 1033 & 1,8-cinéole & 50,00 & 21,00 & 31,20 & 48,12 & 46,00 & 36,01 & 46.20 \\
\hline 1062 & $\gamma$-terpinène & 1,00 & 3,30 & 10,03 & 3,11 & 2,12 & 1,07 & 1,20 \\
\hline 1113 & Fenchol (endo) & 0,30 & 3,85 & - & 0,70 & 0,20 & 0,13 & 0,10 \\
\hline 1139 & Trans-pinocarvéol & 1,50 & 6,64 & - & 1,50 & 1,80 & 4,23 & 3,56 \\
\hline 1163 & Pinocarvone & 0,50 & 2,84 & 0,38 & 0,41 & 0,5 & 1,37 & 1,09 \\
\hline 1177 & Cryptone & - & - & 4,60 & - & - & 2,60 & 1,20 \\
\hline 1189 & $\alpha$-terpinéol & 4,58 & 4,94 & - & 4,64 & 4,40 & 5,38 & 3,80 \\
\hline 1242 & Carvone & 4,60 & 0,18 & - & 1,20 & $\mathbf{0 , 2 3}$ & 1,31 & 4,48 \\
\hline 1439 & Aromadendrène & 0,52 & 0,05 & 4,01 & 0,42 & 0,30 & 0,60 & 0,65 \\
\hline 1462 & Allo-aromadendrène & 0,40 & - & 3,01 & 0,36 & 0,25 & 0,45 & 0,42 \\
\hline 1583 & Globulol & 0,94 & 5,34 & - & 2,50 & 3,30 & 0,90 & 0,44 \\
\hline
\end{tabular}

$\boldsymbol{E} . \boldsymbol{c a} \times \boldsymbol{E} . \boldsymbol{g r}:$ E. camaldulensis $\times$ E. grandis, $\boldsymbol{E} . \boldsymbol{g} \boldsymbol{r} \times \boldsymbol{E} . \boldsymbol{c a}:$ E. grandis $\times \boldsymbol{E}$. camaldulensis, $\boldsymbol{E} . \boldsymbol{c a} \times \boldsymbol{E} . \boldsymbol{t e}:$ E. camaldulensis $\times$ E. tereticornis, $\boldsymbol{E}$. te $\times \boldsymbol{E} . \boldsymbol{c a}:$ E. tereticornis $\times$ E. camaldulensis.

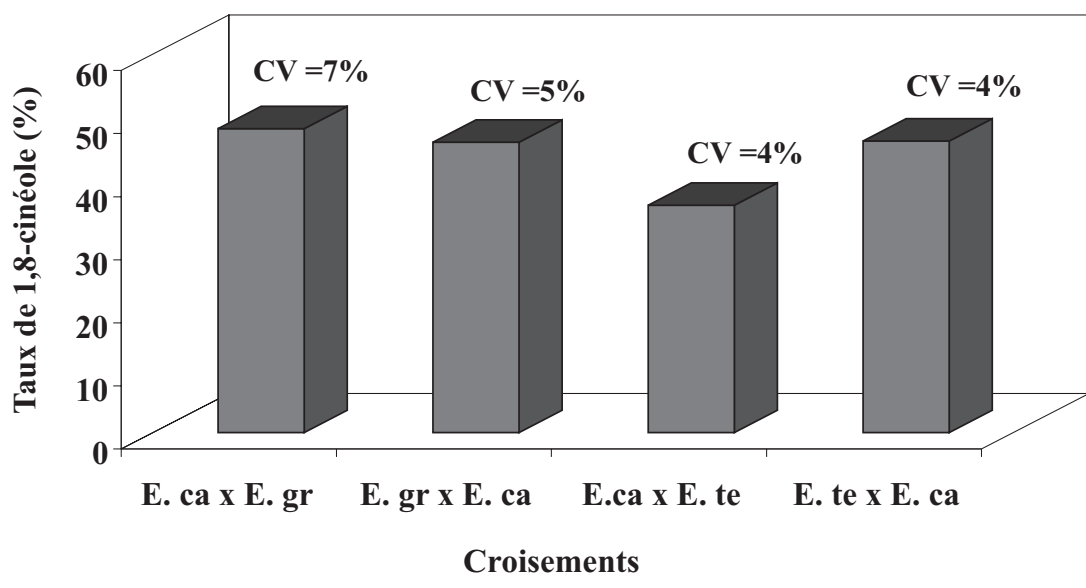

Figure 1. L'influence du croisement et du croisement réciproque sur le taux de 1,8cinéole (CV : coefficient de variation, $E$. ca $\times$ E. $g r:$ E. camaldulensis $\times E$. grandis, E. $g r \times E$. ca $:$ E. grandis $\times E$. camaldulensis, E. ca $\times$ E. te : E. camaldulensis $\times$ E. tereticornis, E. te $\times$ E.ca: E. tereticornis $\times$ E. camaldulensis). 
Tableau V. Analyse de variance à un seul critère de classification (effet des espèces et leurs hybrides sur le taux de 1,8-cinéole des huiles essentielles).

\begin{tabular}{lllll}
\hline Source & Somme des carrés des écarts & Degré de liberté & Carrés moyens & F \\
\hline Espèces & 2777,78 & 6 & 462,96 & $70,19^{* * *}$ \\
Erreur & 138,50 & 21 & 6,59 & \\
\hline
\end{tabular}

$* * *$ : hautement significatif.

fois plus élevées que celle obtenue avec les huiles essentielles des feuilles de l'espèce parentale E. grandis $(21 \%)$ et légèrement inférieures à celle qui est détectée dans les huiles essentielles de l'espèce parentale $E$. $c a$ maldulensis (tableau IV). Par contre les huiles essentielles des échantillons du croisement $E$. camaldulensis $\times$ E. tereticornis, avec un taux de 1,8-cinéole d'environ $36 \%$, légèrement supérieur à celui des feuilles d' $E$. tereticornis $(31,2 \%)$ présentent un taux sensiblement inférieur à celui des échantillons d'E. camaldulensis $(50 \%)$. Ce taux est plus élevé (46\%) lorsque E. camaldulensis intervient comme parent mâle dans le croisement réciproque E. tereticornis $\times E$. camaldulensis. Ce taux est identique à celui de la combinaison $E$. grandis $\times$ camaldulensis (tableau IV et figure 1).

Pour l' $\alpha$-pinène, quelque soit le sens du croisement, le taux de ce produit est bien influencé par la présence d'E. tereticornis (tableau IV). Le taux de p-cymène présente des variations importantes dans les combinaisons entre E. grandis et E. camaldulensis selon que le premier intervient comme parent mâle $(18,64 \%)$ ou femelle $(8,50 \%)$. En ce qui concerne le cryptone qui est une caractéristique d' E. tereticornis, les hybrides ont fournis un taux inférieurs $(2,6$ et $1,2 \%)$ à celui de l'espèce parentale $(4,6 \%)$.

Le carvone, composé principalement présent dans les huiles essentielles d'E. camaldulensis (4,6\%), diminue dans toutes les combinaisons sauf lorsque l'E. camaldulensis intervient comme parent mâle dans la combinaison avec l'E. tereticornis.

Pour le globulol, l'apport du parent E. grandis quant à l'amélioration du taux de ce produit dans les huiles essentielles des hybrides est important, soit 2,5 et $3,3 \%$ pour les hybrides contre 0,44 et $5,34 \%$ respectivement pour les huiles essentielles des espèces parentales $E$. $c a$ maldulensis et E. grandis (tableau IV). Les autres composés présentent une faible variation d'une combinaison à l'autre quelque soit le sens du croisement (tableau IV).

\section{CONCLUSION}

La détermination de la teneur et de la composition chimique des échantillons des huiles essentielles de la biomasse foliaire des eucalyptus étudiés a permis, non seulement d'étudier l'effet de l'hybridation interspécifique, mais aussi de détecter l'influence du sens du croisement sur le taux des constituants majoritaires. Cette hybridation n'a pas d'effet sur la biosynthèse de nouveaux produits.

Quelque soit le sens du croisement, les différentes combinaisons hybrides ne permettent malheureusement pas une amélioration des teneurs en huiles essentielles et des taux de leurs principaux constituants par rapport aux meilleures espèces parentales. Cependant, un effet du sens du croisement sur ces facteurs a été noté, ce qui pourrait inciter à effectuer d'autres travaux de recherche sur l'hérédité de ces caractères, en vue de produire des hybrides susceptibles de fournir les composés recherchés par les industries utilisatrices. Cette première approche de l'effet de l'hybridation sur la teneur et la composition chimique des huiles essentielles des eucalyptus mérite d'être étendue à d'autres combinaisons, avec un plus grand nombre d'observations. Des combinaisons d' $E$. camaldulensis, espèce adaptée aux conditions écologiques et édaphiques difficiles du pays, avec d'autres espèces présentant des teneurs en huiles essentielles élevées et des taux de 1,8-cinéole importants (E. globulus par exemple), pourraient constituer la base d'autres études et observations.

Remerciements : Au terme de ce travail, nous tenons à remercier pour leurs collaborations : Mr. F. Saltron, expert chimiste au laboratoire interrégional de Marseille (France), M. Aberchane du Centre National de la Recherche Forestière (Maroc), MM. S.M. El Youssfi et A. Chakour du Centre National d'Amélioration Génétique des Plants Forestiers (Maroc). 


\section{REFERENCES}

[1] AFNOR, Révision de la norme ISO/CD 770 « Huile essentielle d'E. globulus » (T75 A-Doc 992), 1995.

[2] Artigues R., Lepoutre B., Contribution à la connaissance de l'écologie de l'E. camaldulensis dans les sols sableux de la forêt de la Mamora, Ann. Rech. For. Maroc 11 (1970) 299-327.

[3] Boland D.J., Brophy J., House A.P.N., Eucalyptus leaf oils. Use, chemistry, distillation and marketing, Inkata Press, Melbourne-Sidney, 1991, $252 \mathrm{p}$.

[4] Chalchat J.C., Muhayimana A., Habimana J.L., Chabard J.L., Aromatic plants of Rwanda. II. Chemical composition of essential oils of ten Eucalyptus species growing in Ruhande arboretum, Butare, Rwanda, J. Ess. Oil. Res. 9 (1997) 159-165.

[5] Chaperon H., Amélioration génétique des Eucalyptus au Congo Brazzaville, in: Troisième consultation mondiale sur l'amélioration des arbres forestiers, Canberra, 1976.

[6] Clevenger J.F., Apparatus for the determination of volatile oil, J. Am. Pharm. Assoc. 17 (1928) 336-341.

[7] El Youssfi S.M., Marien J.N., Chakour A., L'amélioration génétique des eucalyptus au Maroc, Actes du séminaire sur l'amélioration, la conservation et l'utilisation des ressources génétiques forestières marocaines, ENFI, 08-10 Mai 1997 (1997) pp. 104-124.

[8] Farah A., Fechtal M., Zrira S., Chaouch A., Les huiles essentielles des eucalyptus hybrides naturels au Maroc. Minbar Al Jamiaâ : Actes du Colloque International sur les Substances Naturelles, 25-26 avril 1997, Méknès, Maroc (1999) pp. 91-97.

[9] GTZ, Les maladies et les dommages affectant les principales essences forestières au Maroc. Coopération maroco-allemande. Projet de protection des forêts, 1990, 92 p.

[10] M'Hirit O., Sbay H., El Alami S.L., Kerrouani H., Bilan de la recherche en matière de génétique forestière au Maroc. Actes du séminaire sur l'amélioration, la conservation et l'utili- sation des ressources génétiques forestières marocaines. ENFI, 8-10 Mai 1997 (1997) pp. 1-29.

[11] Malanda Kiyabou G., Les eucalyptus du Congo : variations inter et intraspécifique du rendement et de la composition chimique de leurs huiles essentielles, Thèse de doctorat, Université Montpellier 2, France, 1992.

[12] MCEF, Ministère Chargé des Eaux et Forêts, Maroc. Bilan des reboisements de la campagne 96-97, 1996, 12 p.

[13] Menut C., Lamaty G., Malanda Kiyabou G., Bessière J.M., Aromatic Plants of tropical Africa. VIII Individual selection of eucalyptus for essential oil production in the Congo, J. Essent. Oil. Res. 4 (1992) 427-432.

[14] Mesbah H., Apport de l'hybridation interspécifique à l'amélioration génétique des eucalyptus au Maroc, Ann. Rech. For. Maroc 28 (1995) 56-71.

[15] Silvestre A.J.D., Cavaleiro J.A.S., Delmond B., Filliatree C., Bourgeois G., The essential oils of eucalyptus globulus labill, From Portugal, Flaowr Frgr. J. 9 (1994) 51-53.

[16] Simmons D., Parsons R.F., Analysis of a hybrid Swarm involving Eucalyptus crenulata and ovata using oils and morphology, Biochem. Syst. Ecolo. 4 (1976) 97-101.

[17] Small B.E.J., The Australian Eucalyptus oil industry an overview, Aust. For. 44 (1981) 170-177.

[18] Wright J.W., Aspects génétiques de l'amélioration des arbres forestiers, FAO, Rome, 1963, 431 p.

[19] Zrira S., Étude des huiles essentielles des Eucalyptus acclimatés au Maroc, Thèse de doctorat es-sciences agronomique. I.A.V. Hassan II. Rabat, Maroc, 1992, 180 p.

[20] Zrira S., Benjilali B., Seasonal changes in volatile oil and cineole contents of five eucalyptus species growing in Morocco, J. Ess. Oil. Res. 8 (1996) 19-24.

[21] Zrira S., Benjilali B., Fechtal M., Richard H., Essential oils of twenty seven eucalyptus species grown in Morocco, J. Ess. Oil. Res. 4 (1992) 259-264. 